\title{
DISEÑO DE LA AUTOMATIZACIÓN DE LA CÁMARA CLIMATIZADA PARA PRUEBAS DE EQUIPOS Y MATERIALES
}

DESIGN OF THE AUTOMATION OF THE AIR-CONDITIONED CHAMBER FOR EQUIPMENT AND MATERIAL TESTING

\author{
María Paula Soto Osorio* \\ Juan Camilo Silva García** \\ Miguel Morales Granados ${ }^{\dagger}$ \\ Carlos Urrego Rodríguez ${ }^{\ddagger}$ \\ FUNDACIÓN UNIVERSIDAD DE AMÉRICA
}

Recibido: 2 de septiembre de 2018 Aceptado: 24 de junio de 2019

DOI: https://doi.org/10.29097/23461098.309

\section{Resumen}

El artículo describe los sistemas de instrumentación, control y supervisión determinados mediante la evaluación de los requerimientos de instrumentación de la ingeniería básica de la cámara climatizada del anteproyecto de Reglamento Técnico de Etiquetado, RETIQ, (2014) y las Normas Técnicas Colombianas 5310 (Instituto Colombiano de Normas Técnicas [ICONTEC], 2004), y 5891 (ICONTEC, 2011). Se aplicó un modelo matemático para hallar parámetros de diseño relacionados con la trasferencia de calor, se eligieron los equipos y, finalmente, se simuló el diseño de automatización en el software de instrumentación virtual, LabVIEW, el cual unifica los sistemas, el modelo matemático y la instrumentación seleccionada. El resultado de esta simulación permitió analizar el comportamiento de la cámara climatizada si usara el diseño de la automatización, pues cumple con los requerimientos nombrados y funciona controlando condiciones atmosféricas específicas para llevar a cabo pruebas de consumo energético a equipos de refrigeración y materiales.

Palabras clave: diseño, automatización, cámara climatizada, sistemas.

\section{Abstract}

The article describes the instrumentation, control, and supervision systems determined by evaluating the instrumentation requirements of the basic engineering of the airconditioned chamber of the Technical Regulations of Labelling. RETIQ (Ministry of Mines and Energy [MINMINAS in its Spanish acronym], 2014), and Colombian Technical Standards 5310 (Colombian Institute of Technical Standards [ICONTEC in its Spa-

\footnotetext{
* Estudiante de Ingeniería Mecánica. Grupo de Investigación en Térmicas y Fluidos, GITF.

** Estudiante de Ingeniería Mecánica. Grupo de Investigación en Térmicas y Fluidos, GITF.

† Ingeniero Mecánico. Especialista en Automatización. Semillero de Automatización, Grupo de Investigación en Térmicas y fluidos, GITF. ⿶ miguel.morales@profesores.uamerica.edu.co

‡ Ingeniero Mecánico. Semillero de Automatización, Grupo de Investigación en Térmicas y Fluidos, GITF.
} 
nish acronym], 2004), and 5891 (ICONTEC, 2011). A mathematical model was applied to find design parameters related to heat transfer, the equipment that makes up the mentioned systems was chosen, and, finally, the automation design was simulated in the virtual instrumentation software, LabVIEW, which unifies the systems, the mathematical model, and the selected instrumentation. The result of this simulation helped to analyse the behaviour of the air-conditioned chamber if the automation design was used since it meets the mentioned requirements and works controlling specific atmospheric conditions to conduct energy consumption tests on cooling devices and materials.

Keywords: design, automation, air-conditioned chamber, systems.

\section{INTRODUCCIÓN}

El principal objetivo del proyecto fue aportar a la industria de refrigeración un diseño de automatización de una cámara climatizada perteneciente a una entidad de tercera parte como la Universidad de América, para generar condiciones de atmósfera controlada con resultados confiables y garantizados, y realizar pruebas energéticas requeridas por el RETIQ a equipos de refrigeración y materiales en diferentes contextos operacionales. Las pruebas de consumo energético que se realizan en la cámara climatizada a equipos de refrigeración necesitan obtener una certificación confiable que cumpla con los parámetros establecidos por los Ministerios de Minas y Energía y de Comercio, Industria y Turismo. El fin de las pruebas es validar la eficiencia energética de los equipos de refrigeración importados para así ser comercializados legalmente en el país. Los objetivos específicos que se plantearon para alcanzar el objetivo principal fueron los siguientes:

- Evaluar los requerimientos de instrumentación y control de la ingeniería básica de la cámara climatizada.

- Seleccionar los equipos necesarios para los sistemas de instrumentación y control de la cámara climatizada.

- Diseñar el sistema de control y supervisión mediante software de instrumentación virtual.

El alcance del proyecto se focalizó en seleccionar los instrumentos y diseñar los sistemas de control y supervisión basados en la ingeniería básica del proyecto previo "Diseño de una cámara climatizada, automatizada, con manejo de información por telemetría y condiciones operativas controladas para pruebas de equipos y materiales”, realizado por Johan Andrés Muñoz Guatibonza (2018). Como límite se estableció que no se construirían ni se instalarían los sistemas desarrollados en el proyecto, tampoco se consideró la fabricación de un prototipo; aunque se efectuó la simulación que ofrece el software de programación virtual del proyecto, finalmente, el tipo de control empleado fue On-Off.

\section{METODOLOGÍA}

La metodología se basó en cumplir los objetivos específicos planteados, desarrollando paso a paso distintas tareas. En primera instancia se caracterizaron los elementos y 
equipos principales que conforman la cámara climatizada, así como los sistemas de refrigeración, calefacción y manejo de aire, con la intención de tener en cuenta el funcionamiento e importancia de cada uno en un sistema global. Posteriormente, se efectuó una evaluación de los requerimientos de instrumentación y control a la cámara desde su ingeniería básica hasta las normativas que rigen el tipo de ensayos para realizar en el laboratorio (mediante la cámara climatizada). Se investigaron prioritariamente requerimientos relacionados con la instrumentación en cuatro secciones, la primera trató las instalaciones de cámara climatizada; la segunda, los equipos de climatización; la tercera, los ensayos de consumo energético para equipos de refrigeración doméstica y comercial, y la última, las pruebas de materiales. Luego de la evaluación por secciones, se procedió a ejecutar un modelo matemático que permitió determinar diferentes parámetros de diseño relacionados con la transferencia de calor en el sistema de climatización y el tiempo de variación de temperatura en el interior de la cámara. Después se efectuó una selección de instrumentos de medición y control, y una justificación de la estrategia de control seleccionada al inicio de la investigación. Seguidamente, se procedió a seleccionar el software de instrumentación virtual y a diseñar los sistemas de control y supervisión para la generación de condiciones atmosféricas controladas dentro de la cámara en una simulación proporcionada por este software. Tras estas actividades, se realizó un presupuesto con los costos de la ejecución del proyecto, contemplando los diferentes valores involucrados, como el valor de los instrumentos, licencia del software, costos de instalación y mano de obra. Finalmente, se elaboraron los planos correspondientes para la adecuada instalación de los sistemas de instrumentación y control, y una guía de mantenimiento para cuidar la vida útil de los instrumentos seleccionados.

\section{MARCO TEÓRICO}

Cámara climatizada. Es un laboratorio diseñado para generar condiciones de atmósfera controlada, con dos divisiones. El primer laboratorio es para pruebas de equipos de refrigeración doméstico, y el segundo, para pruebas de equipos de refrigeración comercial. Su principio de funcionamiento es la climatización.

Climatización. Es el proceso en el cual el aire se encarga de producir condiciones climáticas en una instalación cerrada por medio de la composición de sus gases, propiedades y de las variables físicas como temperatura, humedad y velocidad de flujo de aire. (Muñoz, 2018).

\section{RESULTADOS}

Caracterización de los elementos. Son los resultados obtenidos en la caracterización de los elementos y equipos de climatización llevaron a entender su funcionamiento y las condiciones de atmósfera que generan luego del proceso de enfriamiento, deshumidificación, calentamiento o humidificación. Durante el proceso de calentamiento y humidificación, en la cámara se usó únicamente el sistema de climatización, el cual está conformado por la unidad manejadora de aire y funciona como se ilustra en la Figura 1: 
Soto Osorio, Silva García, Morales Granados y Urrego Rodríguez

Figura 1

Diagrama de bloques de proceso de calentamiento y humidificación.

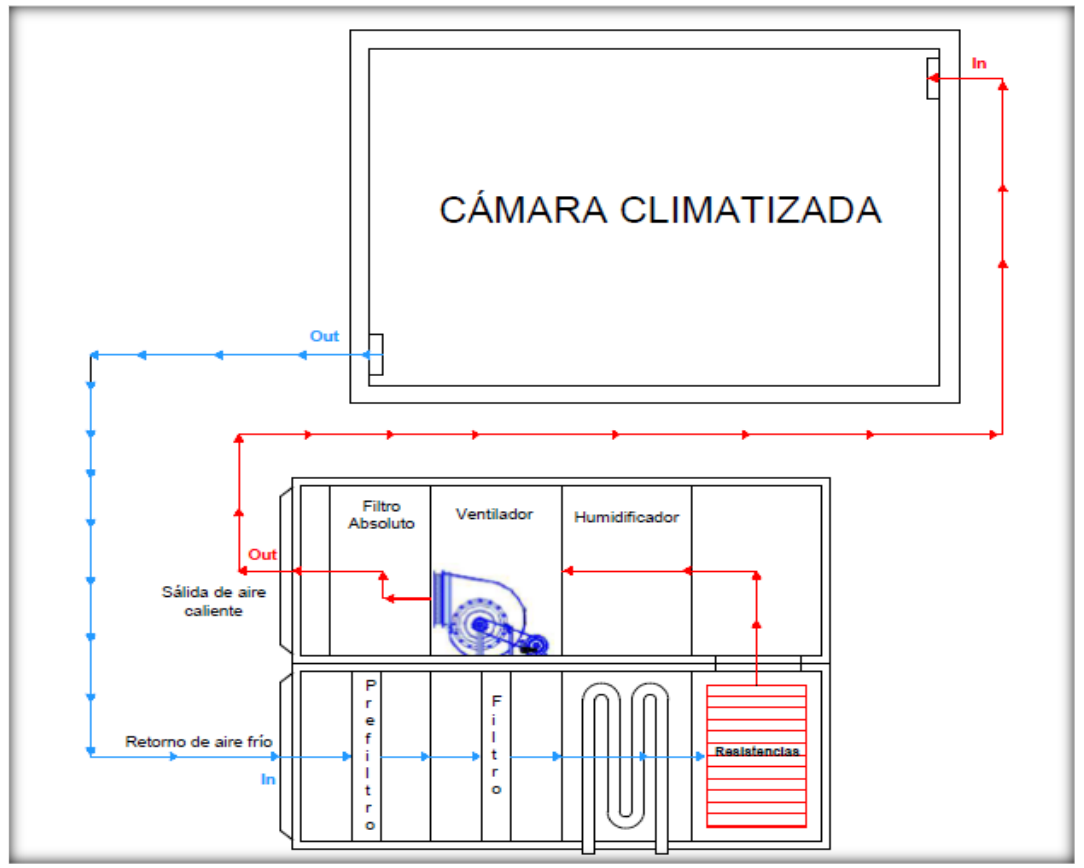

Nota. Esquema tomado de Silva y Soto (2019).

Figura 2

Diagrama de bloques de enfriamiento y deshumidificación

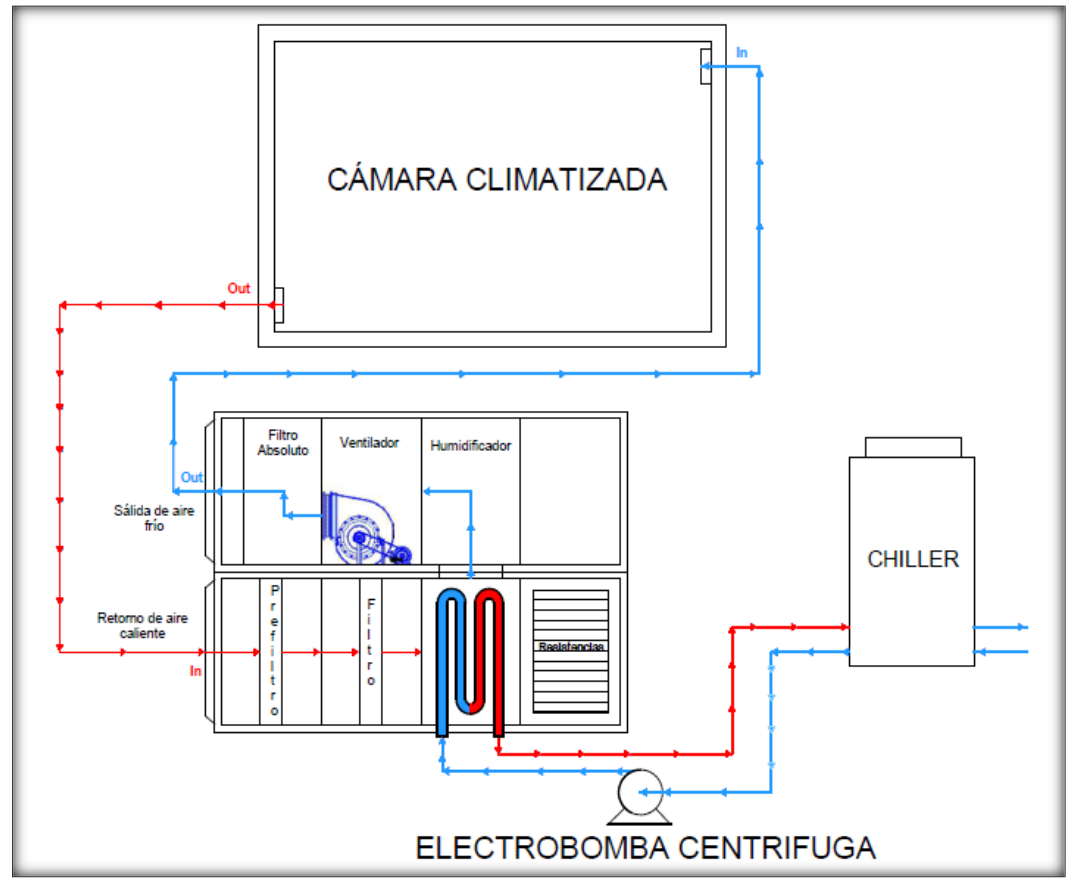

Nota. Esquema tomado de Silva y Soto (2019). 
En el proceso de enfriamiento y deshumidificación, el sistema de refrigeración y climatización trabajan en conjunto; los equipos que lo componen son una electrobomba, una enfriadora tipo chiller, un serpentín de cobre y, por supuesto, la unidad manejadora de aire. En la Figura 2 se muestra el funcionamiento a través de un diagrama de bloques.

\section{Evaluación de requerimientos de instrumentación}

Los resultados obtenidos después de la evaluación de instrumentos en la sección de las instalaciones de la cámara climatizada y los equipos de climatización se resumen a continuación en la Tabla 1 y la Figura 3.

Tabla 1

Resumen de los puntos de medición de la sección 1 y 2

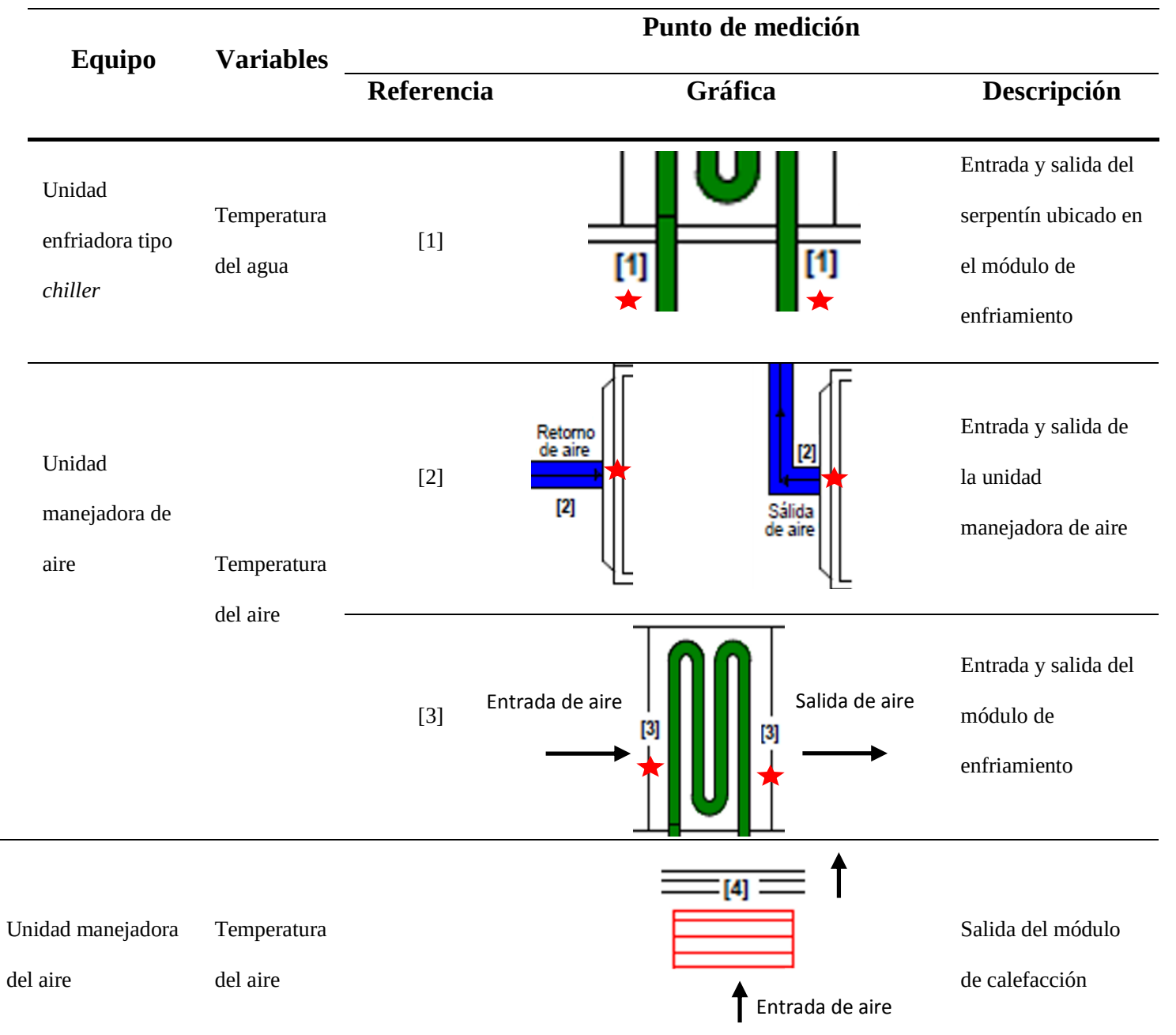

[4] 
[5]

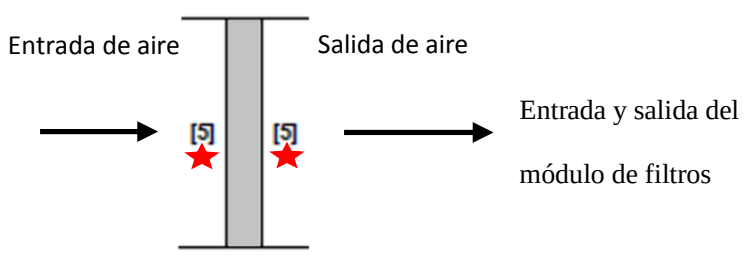

relativa del

Velocidad del

flujo de aire
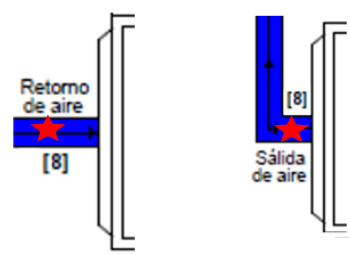

Entrada y salida de

la unidad

manejadora de aire

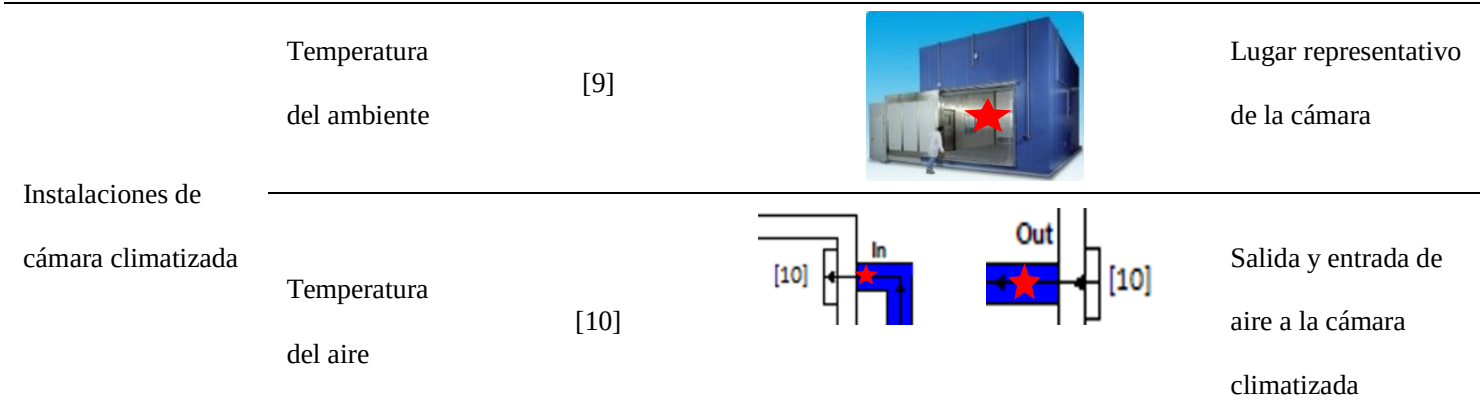

Nota. Esquemas tomados de Silva y Soto (2019).

En la sección de referencia tres, se evaluaron los requerimientos de instrumentación relacionados con los ensayos de consumo energético establecidos en el Reglamento Técnico de Etiquetado. RETIQ (MINMINAS, 2014), y las Normas Técnicas Colombianas 5310 (ICONTEC, 2004), y 5891 (ICONTEC, 2011). Los resultados obtenidos se sintetizaron por laboratorio en las tablas 2 y 3. 
La última sección, no tuvo requerimientos adicionales y con los ya evaluados se pueden llevar a cabo las pruebas a materiales y equipos de refrigeración

Figura 3

Posición de los instrumentos según la referencia numérica de la sección 1 y 2

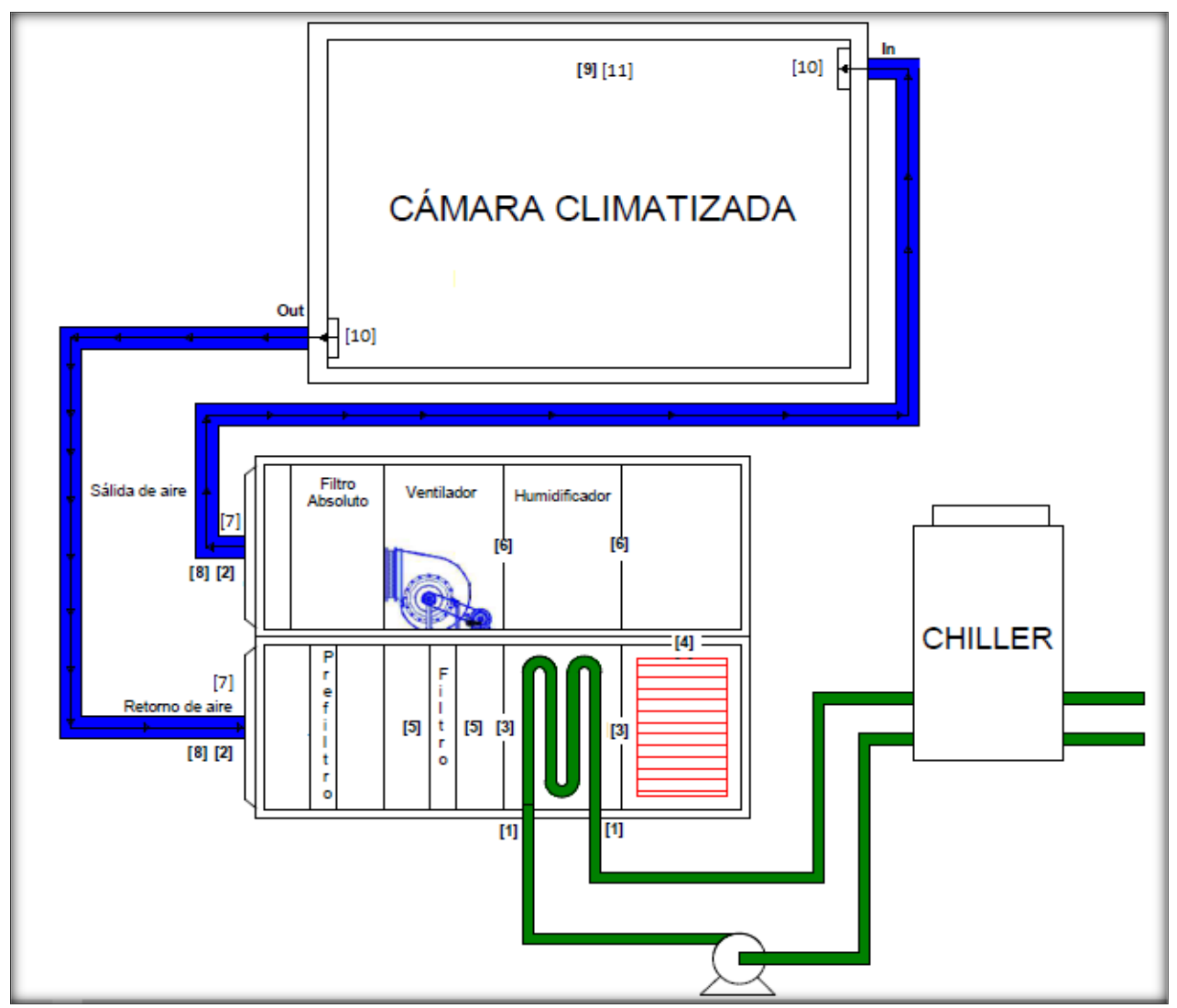

Nota. Esquema tomado de Silva y Soto (2019). 
Tabla 2

Síntesis de requerimientos de instrumentación en el laboratorio de pruebas de equipos domésticos

\begin{tabular}{|c|c|c|c|c|c|c|c|c|}
\hline $\begin{array}{l}\text { Tipo de } \\
\text { ensayo }\end{array}$ & $\begin{array}{c}\text { Características } \\
\text { de la cámara } \\
\text { climatizada }\end{array}$ & $\begin{array}{l}\text { Suministro } \\
\text { de energía }\end{array}$ & Variables & $\begin{array}{l}\text { Cantidad de } \\
\text { instrumentos }\end{array}$ & $\begin{array}{c}\text { Intervalo } \\
\text { de medida }\end{array}$ & $\begin{array}{l}\text { Margen de } \\
\text { error }\end{array}$ & $\begin{array}{c}\text { Incertidumbre } \\
\text { máxima }\end{array}$ & $\begin{array}{l}\text { Intervalo } \\
\text { de medición }\end{array}$ \\
\hline \multirow{4}{*}{ Doméstico } & \multirow{3}{*}{$\begin{array}{c}4 \text { m de ancho, } \\
10 \text { m de largo, } 3 \\
\text { m de altura }\end{array}$} & \multirow{4}{*}{$115 \mathrm{~V} \pm 2 \mathrm{~V}$} & $\begin{array}{c}\text { Temperatu } \\
\text { ra }\end{array}$ & $\begin{array}{l}6 \text { / unid. } \\
48 \text { en total }\end{array}$ & $0{ }^{\circ} \mathrm{C}$ a $50^{\circ} \mathrm{C}$ & $\pm 2{ }^{\circ} \mathrm{C}$ & $\pm 0,5^{\circ} \mathrm{C}$ & $\mathrm{R} \leq 60 \mathrm{~s}$ \\
\hline & & & Humedad & 2 & $45 \%$ a $98 \%$ & $\pm 1 \%$ & $\pm 0,3^{\circ} \mathrm{C}$ & $\mathrm{R} \leq 60 \mathrm{~s}$ \\
\hline & & & $\begin{array}{l}\text { V. flujo de } \\
\text { aire }\end{array}$ & $\begin{array}{l}3 \text { / unid. } \\
24 \text { en total }\end{array}$ & $\begin{array}{c}0,10 \mathrm{~m} / \mathrm{s} \text { a } 5 \\
\mathrm{~m} / \mathrm{s}\end{array}$ & $\pm 5 \%$ & $\pm 0,5 \mathrm{~m} / \mathrm{s}$ & $\mathrm{R} \leq 60 \mathrm{~s}$ \\
\hline & $\begin{array}{c}\text { Carga térmica } \\
10 \mathrm{TR}\end{array}$ & & $\begin{array}{l}\text { Consumo } \\
\text { de energía }\end{array}$ & $\begin{array}{l}1 \text { / unid. } \\
8 \text { en total }\end{array}$ & $\begin{array}{c}\geq \\
0,001 \mathrm{Kw} / \mathrm{h}\end{array}$ & $\pm 1 \%$ & $\longrightarrow$ & $24 \mathrm{~h}$ \\
\hline
\end{tabular}


Tabla 3

Síntesis de los requerimientos de instrumentación del laboratorio para pruebas de equipos comerciales

\begin{tabular}{|c|c|c|c|c|c|c|c|c|}
\hline $\begin{array}{l}\text { Tipo de } \\
\text { ensayo }\end{array}$ & $\begin{array}{l}\text { Dimensiones de } \\
\text { la cámara } \\
\text { climatizada }\end{array}$ & $\begin{array}{l}\text { Suministro } \\
\text { de energía }\end{array}$ & Variables & $\begin{array}{l}\text { Cantidad de } \\
\text { instrumentos }\end{array}$ & $\begin{array}{l}\text { Intervalo de } \\
\text { medida }\end{array}$ & $\begin{array}{l}\text { Margen } \\
\text { de error }\end{array}$ & $\begin{array}{c}\text { Incertidumbre } \\
\text { máxima }\end{array}$ & $\begin{array}{l}\text { Intervalo } \\
\text { de } \\
\text { medición }\end{array}$ \\
\hline \multirow{4}{*}{ Comercial } & $\begin{array}{l}6 \mathrm{~m} \text { de ancho, } 10 \\
\mathrm{~m} \text { de largo, } 3 \mathrm{~m}\end{array}$ & \multirow{4}{*}{$230 \mathrm{~V} \pm 2 \mathrm{~V}$} & Temperatura & $\begin{array}{c}\text { Varía según } \\
\text { dimensiones de } \\
\text { los equipos }\end{array}$ & $0{ }^{\circ} \mathrm{C}$ a $50{ }^{\circ} \mathrm{C}$ & $\pm 2^{\circ} \mathrm{C}$ & $\pm 1,5^{\circ} \mathrm{C}$ & $\mathrm{R} \leq 60 \mathrm{~s}$ \\
\hline & de altura & & Humedad & 2 & $50 \%$ а $99 \%$ & $\pm 2 \%$ & $\pm 0,3^{\circ} \mathrm{C}$ & $\mathrm{R} \leq 60 \mathrm{~s}$ \\
\hline & Carga & & $\begin{array}{l}\text { V. flujo de } \\
\text { aire }\end{array}$ & $\begin{array}{l}3 \text { por equipo/ } 9 \\
\text { en total }\end{array}$ & $\begin{array}{c}0,10 \mathrm{~m} / \mathrm{s} \text { a } 5 \\
\mathrm{~m} / \mathrm{s}\end{array}$ & $\pm 5 \%$ & $\pm 0,5 \mathrm{~m} / \mathrm{s}$ & $\mathrm{R} \leq 60 \mathrm{~s}$ \\
\hline & térmica: 15 TR & & $\begin{array}{c}\text { Consumo de } \\
\text { energía }\end{array}$ & $\begin{array}{c}1 \text { por equipo/ } 3 \\
\text { en total }\end{array}$ & $\geq 0,001 \mathrm{Kw} / \mathrm{h}$ & $\pm 1 \%$ & - & $24 \mathrm{~h}$ \\
\hline
\end{tabular}




\section{Modelo matemático}

El modelo matemático se realizó determinando dos sistemas principales, el primero se relacionaba con los equipos de climatización, y el segundo, con las instalaciones de la cámara climatizada. Entonces para el primer sistema, se estableció que solamente se presentan dos posibles combinaciones de procesos con sus respectivos elementos de funcionamiento, tal como se ve en las Figuras 4 y 5.

Figura 4

Calentamiento y humidificación

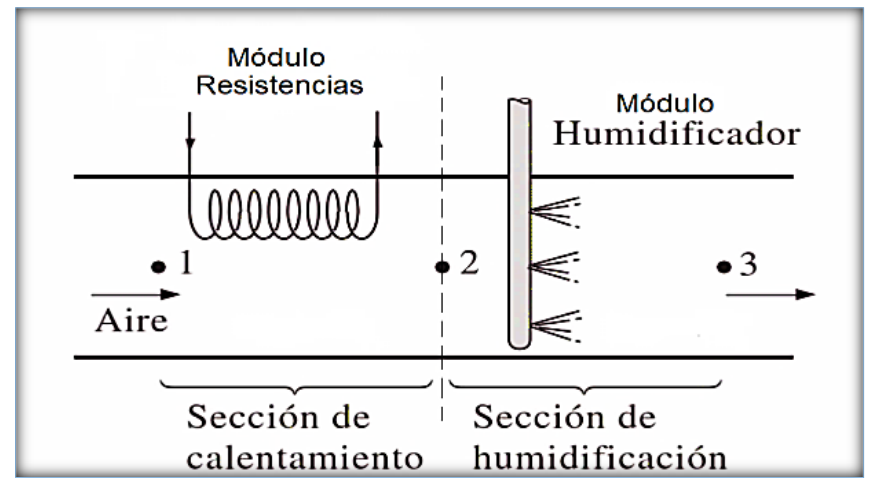

Nota. Esquema tomado de Cengel (2017).

Figura 5

Enfriamiento, deshumidificación y calentamiento

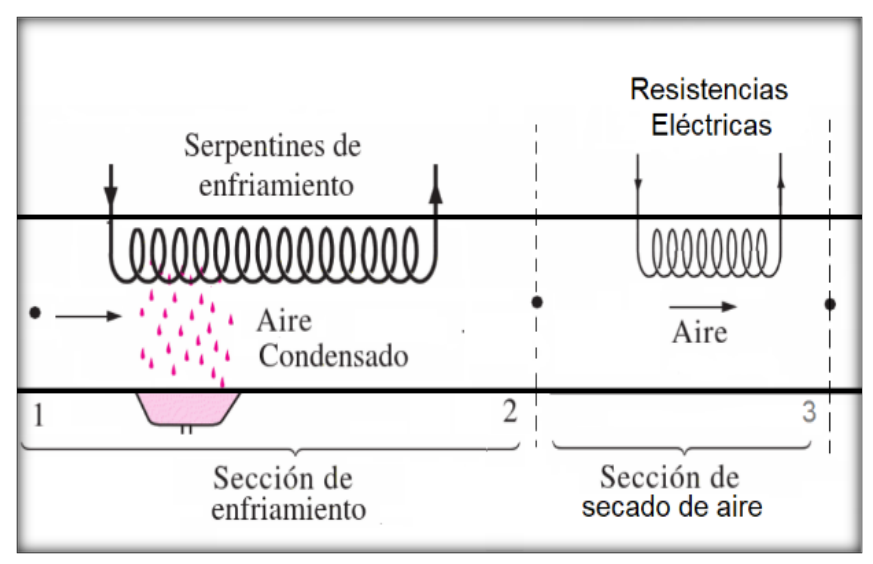

Nota. Esquema tomado de Cengel (2017).

Las deducciones establecidas en las posibles combinaciones, anteriores son:

- Proceso de flujo estable. La masa de aire circulante se mantiene constante a través de los módulos con respecto al tiempo. 
- Cada módulo es un volumen de control.

- El contenido de energía total de cada uno de los volúmenes de control permanece constante, por lo tanto, la variación de energía total es cero (Cengel, 2017).

- El aire seco y el vapor son gases ideales.

- El calor específico para la aplicación de climatización es de 1,005 KJ / Kg. ${ }^{\circ} \mathrm{C}$ (Cengel, 2017).

- La energía cinética es constante, puesto que la velocidad es constante durante los procesos.

- La energía potencial no presenta variaciones, puesto que la altura se mantiene sin cambios significativos en los equipos de climatización.

- En el módulo de resistencias eléctricas, la tasa de calor de entrada y salida es cero, debido a que el módulo es totalmente aislado; no obstante, sí existe trabajo eléctrico generado por las resistencias en las dos combinaciones, como se observa en las siguientes ecuaciones.

Ecuación 1. Balance de energía de volumen de control del módulo de calefacción en la combinación de procesos, calentamiento y humidificación (Cengel, 2017).

$$
\dot{\mathrm{W}}_{E n t}=\dot{\mathrm{m}}_{a}\left(h_{2}-h_{1}\right)
$$

Ecuación 2. Balance de energía de volumen de control del módulo de calefacción en la combinación de procesos, enfriamiento, deshumidificación y calentamiento (Cengel 2017).

$$
\dot{\mathrm{W}}_{\text {Ent }}=\dot{\mathrm{m}}_{a}\left(h_{3}-h_{2}\right)
$$

En donde:

$\dot{\mathrm{W}}_{\text {Ent }}=$ es el trabajo eléctrico de las resistencias en $\mathrm{kW}$

$\dot{m}_{a}=$ es el flujo másico del aire seco en $\mathrm{kg} / \mathrm{s}$

$h=$ es la entalpía de cada estado en $\mathrm{kJ} / \mathrm{kg}$

- En el módulo de humidificación, también, se presenta trabajo eléctrico por las resistencias encargadas de calentar el agua con que se humidifica el aire, entonces, el balance resulta de la siguiente forma:

Ecuación 3. Balance de energía de volumen de control del módulo de humidificación en la combinación de procesos calentamiento y humidificación. (Cengel, 2017).

$$
\dot{\mathrm{W}}_{E n t}=\dot{\mathrm{m}}_{a}\left(h_{3}-h_{2}\right)-\dot{\mathrm{m}}_{w} \cdot h_{f}
$$


En donde:

$\dot{\mathrm{W}}_{\text {Ent }}=$ es el trabajo de las resistencias del módulo de humidificación en kW

$\dot{m}_{a}=$ es el flujo másico del aire seco en $\mathrm{kg} / \mathrm{s}$

$h=$ es la entalpía de cada estado en $\mathrm{kJ} / \mathrm{kg}$

$\dot{m}_{w}=$ es el flujo másico del agua de adición en $\mathrm{kg} / \mathrm{s}$

$h_{f}=$ es la entalpía de líquido saturado en $\mathrm{kJ} / \mathrm{kg}$

- En el módulo de enfriamiento, sí se presenta tasa de calor de entrada, debido a que el aire le cede el calor al serpentín de cobre para enfriarse; entonces, el balance de energía resulta como se muestra en la siguiente ecuación.

Ecuación 4. Balance de energía del módulo de enfriamiento (Cengel, 2017).

$$
\overline{\mathrm{Q}}_{E n t}=\dot{\mathrm{m}}_{a}\left(h_{2}-h_{1}\right)-\dot{\mathrm{m}}_{w} \cdot h_{f}
$$

En donde:

$\dot{m}_{a}=$ es el flujo másico del aire seco en $\mathrm{kg} / \mathrm{s}$

$h$ = es la entalpía de cada estado en $\mathrm{kJ} / \mathrm{kg}$

$\dot{m}_{w}=$ es el $\mathrm{l}$ agua que se condensa en el módulo de enfriamiento en kg/s

$h_{f}=$ es la entalpía de líquido saturado $\mathrm{kJ} / \mathrm{kg}$

Por otro lado, las propiedades físicas que se establecieron en el modelo matemático fueron las siguientes:

- El flujo volumétrico en el laboratorio de ensayos de equipos domésticos es 0,15 $\mathrm{m}^{3} / \mathrm{s}$, y para el otro laboratorio es de $0,2 \mathrm{~m}^{3} / \mathrm{s}$. (Barbosa y Gamboa, 2019).

- La presión total de los procesos es la de Bogotá, 560 mmHg (74,66).

- La constante de gas tiene un valor de $0,287 \mathrm{~kJ} / \mathrm{kg}$.

- La presión de saturación del aire atmosférico depende de su temperatura.

- La entalpía de líquido y vapor saturados también depende de la temperatura del aire.

Los posibles escenarios en la combinación de proceso se determinan en las siguientes tablas, por variable física y estado. 
Tabla 4

Resumen de los posibles escenarios considerados en la combinación de calentamiento y humidificación.

\begin{tabular}{|c|c|c|c|}
\hline & \multicolumn{3}{|c|}{ Calentamiento y humidificación } \\
\hline \multirow{2}{*}{ Estado 1 } & Temperatura & Humedad relativa & Humeda especifica \\
\cline { 2 - 3 } & $T_{1}=T_{a m b}$ & $\emptyset_{1}<\emptyset_{3}$ & $\omega_{1}=\frac{0.622 \cdot \emptyset_{1} \cdot P_{g 1}}{P_{T}-\emptyset_{1} \cdot P_{g 1}}$ \\
\cline { 2 - 3 }${ }^{\circ} \mathrm{C}<T_{1}<45^{\circ} \mathrm{C}$ & $45 \% \leq \emptyset_{1} \leq 98 \%$ & $\omega_{1}=\omega_{2}$ \\
\hline \multirow{2}{*}{ Estado 2 } & $T_{2}<T_{3}$ & $\emptyset_{2}=\frac{\omega_{2} \cdot P_{T}}{\left(0.622+\omega_{2}\right) \cdot P_{\Omega 2}} \cdot 100 \%$ & \\
\hline \multirow{2}{*}{ Estado 3 } & $2^{\circ} \mathrm{C} \leq T_{3} \leq 45^{\circ} \mathrm{C}$ & $45 \% \leq \emptyset_{3} \leq 98 \%$ & $\omega_{3}=\frac{0.622 \cdot \emptyset_{3} \cdot P_{g 3}}{P_{T}-\emptyset_{3} \cdot P_{g 3}}$ \\
\hline
\end{tabular}

Nota. Tabla tomada de Silva y Soto (2019).

Tabla 5

Resumen de Resumen de los posibles escenarios considerados en la combinación de enfriamiento, deshumidificación y calentamiento.

\begin{tabular}{|c|c|c|c|}
\hline \multicolumn{4}{|c|}{ Enfriamiento, Deshumidificación y calentamiento } \\
\hline & Temperatura & Humedad relativa & Humeda especifica \\
\hline \multirow{3}{*}{ Estado 1} & $T_{1}=T_{a m b}$ & $\emptyset_{1}=\emptyset_{a m b}$ & \multirow{3}{*}{$\omega_{1}=\frac{0.622 \cdot \emptyset_{1} \cdot P_{g}}{P_{T}-\emptyset_{1} \cdot P_{g 1}}$} \\
\hline & $2^{\circ} \mathrm{C}<T_{1}<45^{\circ} \mathrm{C}$ & $\emptyset_{1}>\emptyset_{3}$ & \\
\hline & $T_{1}>T_{3}$ & $45 \% \leq \emptyset_{1} \leq 98 \%$ & \\
\hline Estado 2 & $T_{2}<T_{3}$ & $\emptyset_{2}=1$ & $\omega_{1}>\omega_{2}$ \\
\hline Estado 3 & $2^{\circ} \mathrm{C} \leq T_{3} \leq 45^{\circ} \mathrm{C}$ & $\emptyset_{3}<75 \%$ & $\omega_{2}=\omega_{3}$ \\
\hline
\end{tabular}

Nota. Tabla tomada de Silva y Soto (2019).

Ahora bien, para el segundo sistema relacionado con las instalaciones de la cámara climatizada el modelo matemático se enfocó en obtener el tiempo de variación de temperatura con el fin de seleccionar correctamente los instrumentos respecto al lapso de reacción esperado.

Para obtener la ecuación que determinó el tiempo de variación en $1{ }^{\circ} \mathrm{C}$ en la cámara climatizada, se analizó el desarrollo de la combinación de procesos, y se obtuvo la siguiente ecuación. 
Ecuación 5. temperatura de equilibrio en las instalaciones de la cámara climatizada (Silva y Soto, 2019).

$$
T_{\text {equilibrio }}=\frac{\left(\frac{10 \cdot \dot{\mathrm{m}}_{a S D}}{\dot{\mathrm{m}}_{a C C}} \cdot T_{2}\right)+T_{1}}{\left(1+\frac{10 \cdot \dot{\mathrm{m}}_{a S D}}{\dot{\mathrm{m}}_{a C C}}\right)}
$$

Los resultados obtenidos para este sistema se detallan en la tabla 6 y la figura 6 .

Tabla 6

Resultado de tiempo de variación en $1{ }^{\circ} \mathrm{C}$.

\begin{tabular}{cc}
\hline Incremento de temperatura $\left({ }^{\circ} \mathbf{C}\right)$ & Tiempo (min) \\
\hline 0 & 0 \\
\hline 1 & 16,899 \\
\hline 2 & 33,798 \\
\hline 3 & 50,697 \\
\hline 4 & 67,596 \\
\hline 5 & 84,496 \\
\hline 6 & 101,395 \\
\hline 7 & 118,294 \\
\hline 8 & 135,193 \\
\hline 9 & 152,093 \\
\hline 10 & 168,992 \\
\hline
\end{tabular}

Nota. Tabla tomada de Silva y Soto (2019). 
Figura 6

Comportamiento de la temperatura con respecto al tiempo

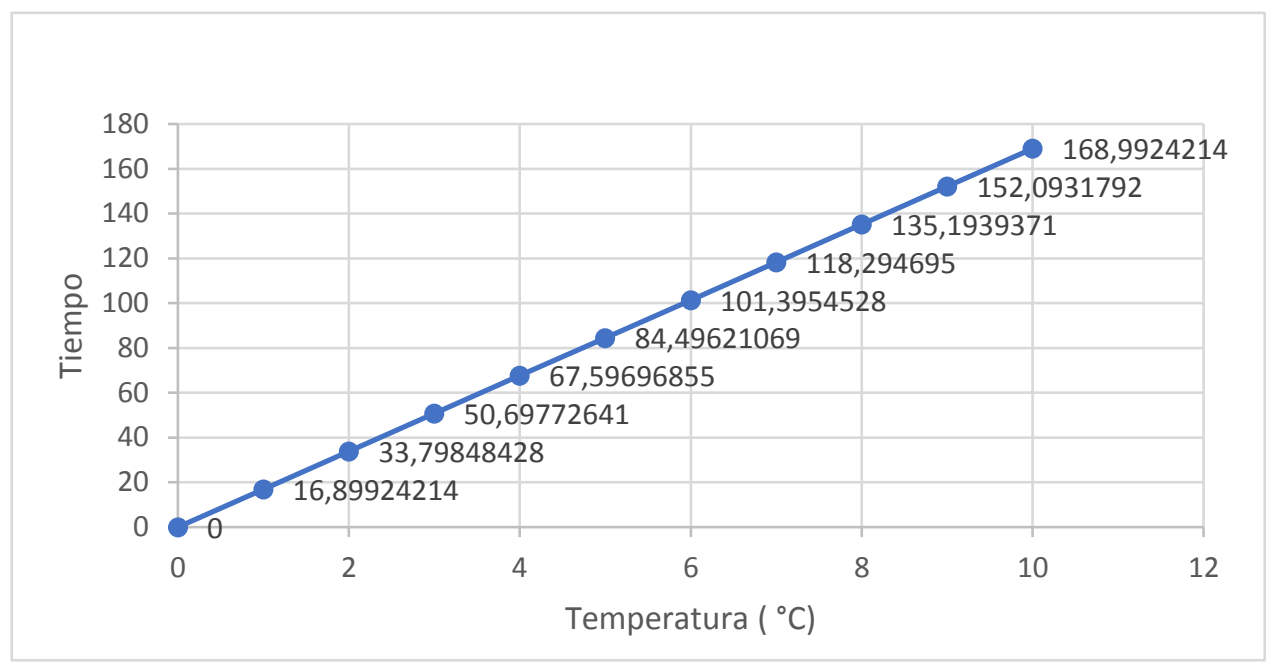

Nota. Figura tomada de Silva y Soto (2019).

\section{Selección de instrumentos}

Una vez determinada la ubicación, la cantidad y el tiempo de variación de temperatura en la cámara climatizada, se seleccionaron los instrumentos para suplir dichas necesidades y se detallaron en la tabla 7.

Tabla 7

Instrumentos seleccionados

\begin{tabular}{ccccc}
\hline $\mathbf{N .}^{\mathbf{0}}$ & Ítems & Proveedor & Cantidad & Imagen \\
\hline 11 & $\begin{array}{c}\text { Sensor de temperatura por } \\
\text { resistencia serie RTD - 646 }\end{array}$ & Dwyer & 93 \\
\hline 2 & Extensión de cable & Dwyer & 96 & 2 \\
\hline 3 & $\begin{array}{c}\text { Transmisor de humedad y } \\
\text { temperatura, serie RHP- } \\
\text { 2W1D }\end{array}$ & Dwyer & \\
\hline
\end{tabular}




\begin{tabular}{|c|c|c|c|c|}
\hline 4 & $\begin{array}{l}\text { Transmisor de velocidad de } \\
\text { aire 641-6 }\end{array}$ & Dwyer & 33 & \\
\hline 5 & $\begin{array}{l}\text { Transmisor de humedad y } \\
\text { temperatura Serie RHP-2D1B }\end{array}$ & Dwyer & 8 & \\
\hline 6 & $\begin{array}{l}\text { Transmisor de velocidad de } \\
\text { aire serie AVU-1-A }\end{array}$ & Dwyer & 6 & \\
\hline 7 & $\begin{array}{l}\text { Válvula esférica de acero } \\
\text { inoxidable NPT de } 3 \text { vías serie } \\
\text { WE31 }\end{array}$ & Dwyer & 2 & \\
\hline 8 & $\begin{array}{l}\text { Interruptor de protección } \\
\text { anticongelante de límite serie } \\
\text { DFS-DA20 }\end{array}$ & Dwyer & 2 & \\
\hline 9 & $\begin{array}{l}\text { Controlador de temperatura } \\
\text { TCN4L-24R }\end{array}$ & Autonics & 6 & 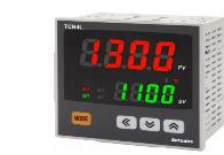 \\
\hline 10 & $\begin{array}{l}\text { Amperímetro digital M4W- } \\
\text { AA-2 }\end{array}$ & Autonics & 11 & 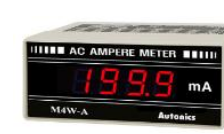 \\
\hline 11 & $\begin{array}{l}\text { Actuador para Dampers serie } \\
\text { DDA11 }\end{array}$ & Dwyer & 22 & \\
\hline 12 & $\begin{array}{l}\text { Sistema Compact Rio cRIO } \\
9032\end{array}$ & $\begin{array}{l}\text { National } \\
\text { Instruments }\end{array}$ & 2 & \\
\hline
\end{tabular}

Nota. Tabla tomada de Silva y Soto (2019).

\section{Selección de software de instrumentación}

La determinación del software de instrumentación virtual se realizó mediante el método de decisión multicriterio scoring, en donde se evidenció que la mejor alternativa era el programa LabVIEW, como se expone en la siguiente tabla de resultados. 
Tabla 8

Resultados del método scoring

\begin{tabular}{lllll}
\hline Criterios & $\begin{array}{l}\text { Ponderación } \\
\text { (Wi) }\end{array}$ & $\begin{array}{l}\text { UniLogic } \\
\text { (ri1) }\end{array}$ & $\begin{array}{l}\text { LabView } \\
\text { (ri2) }\end{array}$ & $\begin{array}{l}\text { LabWindows } \\
\text { (ri3) }\end{array}$ \\
\hline 1. Lenguaje de programación & 30 & 30 & 50 & 30 \\
\hline 2. Comunicación con el sistema & 40 & 20 & 50 & 40 \\
de adquisición de datos & & & & 30 \\
\hline 3. Información en tiempo real & 40 & 30 & 40 & 30 \\
\hline 4. Costo de la licencia & 30 & 30 & 40 & 40 \\
\hline 5. Simulación & 50 & 30 & 50 & 6600 \\
\hline Total & & 5300 & 8800 & \\
\hline
\end{tabular}

Nota. Tabla tomada de Silva y Soto (2019).

\section{Selección de software de instrumentación}

Una vez seleccionado el software virtual, se diseñaron los sistemas de control y supervisión; el sistema de control se conforma por tres lazos de control cerrado y cuatro lazos de control abierto. Los lazos de control cerrados se configuraron para controlar y modificar las condiciones atmosféricas en la cámara climatizada, y los lazos de control abiertos se diseñaron para la adquisición y supervisión de variables de temperatura, humedad y velocidad del aire en los puntos establecidos anteriormente.

El resultado de estos dos sistemas se evidencia en las interfaces de usuario y del proceso; en las Figuras 7 y 8 se ilustran las interfaces por procesos de enfriamiento y deshumidificación o calentamiento y humidificación, en donde estratégicamente se adicionaron alarmas a los parámetros de diseño, con el fin de supervisar desde otra perspectiva el correcto funcionamiento de los sistemas y de los parámetros de operación necesarios.

También, en cada interfaz se ubicó un botón maestro, para definirle al sistema si se necesitan los resultados del laboratorio de pruebas de equipos de refrigeración doméstico o comercial; finalmente, los ledes proporcionaron una visualización simultánea al operario de los equipos o elementos en funcionamiento. 


\section{Soto Osorio, Silva García, Morales Granados y Urrego Rodríguez}

\section{Figura 7}

Interfaz de los procesos de enfriamiento y deshumidificación

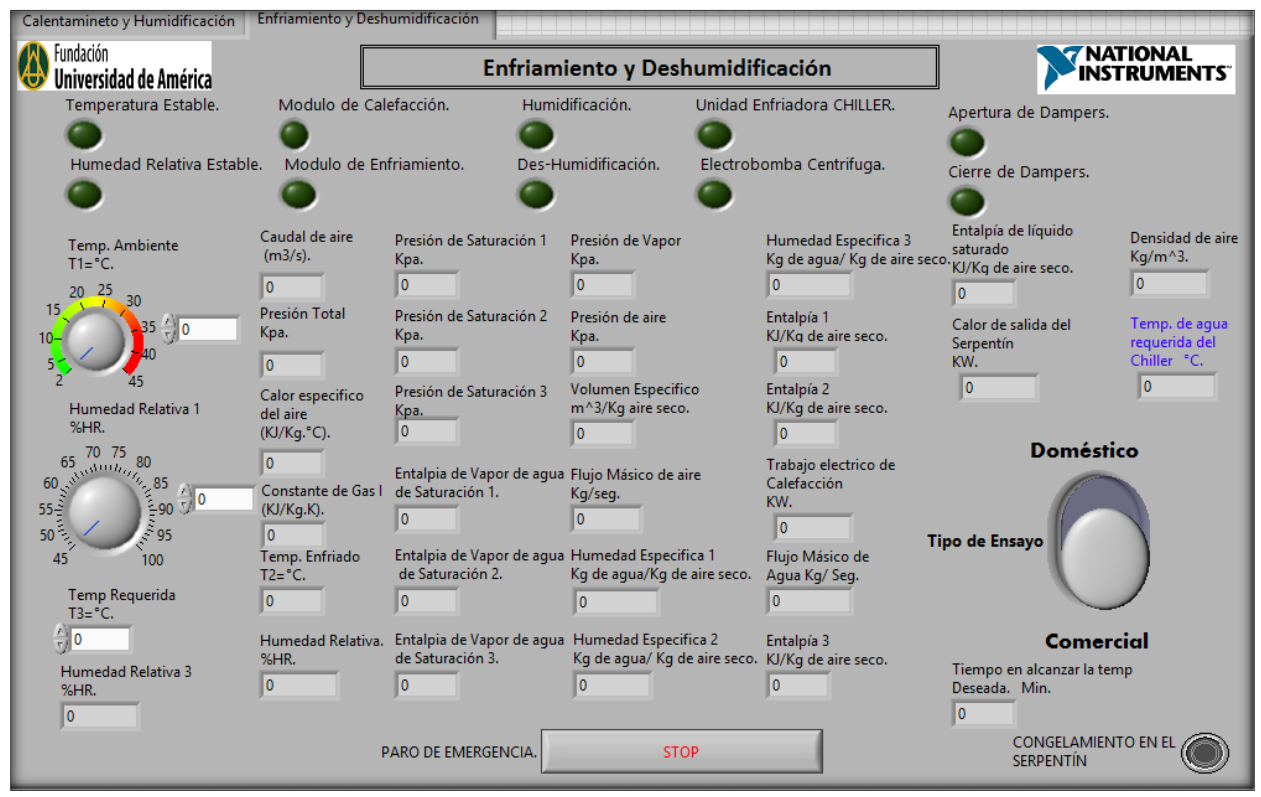

Nota. Imagen tomada de Silva y Soto (2019).

\section{Figura 8}

Interfaz de los procesos de calentamiento y humidificación

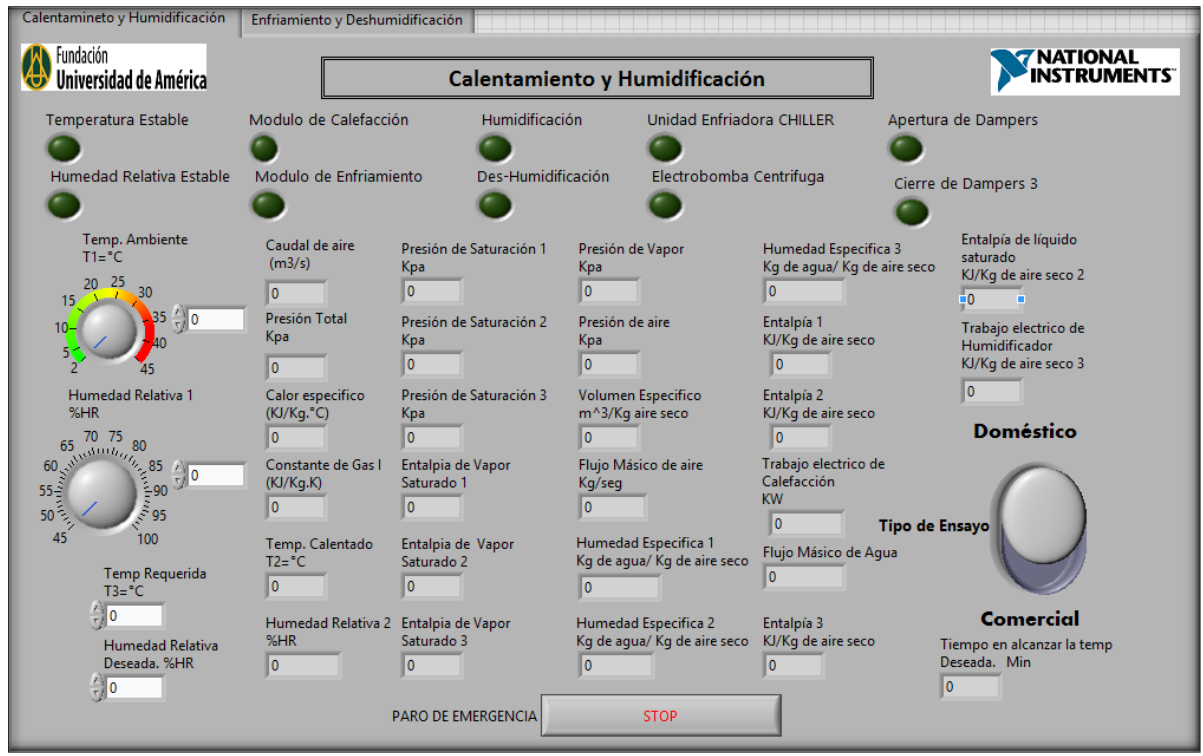

Nota. Imagen tomada de Silva y Soto (2019).

Además, en el sistema de supervisión se programó un cambio de color para cada máquina, con la intención de vigilar si trabajaban correctamente o alguna detuvo su 
funcionamiento. En la Figura 9, se visualiza dicho comportamiento en el programa LabVIEW.

Figura 9

Sistema global en correcto funcionamiento

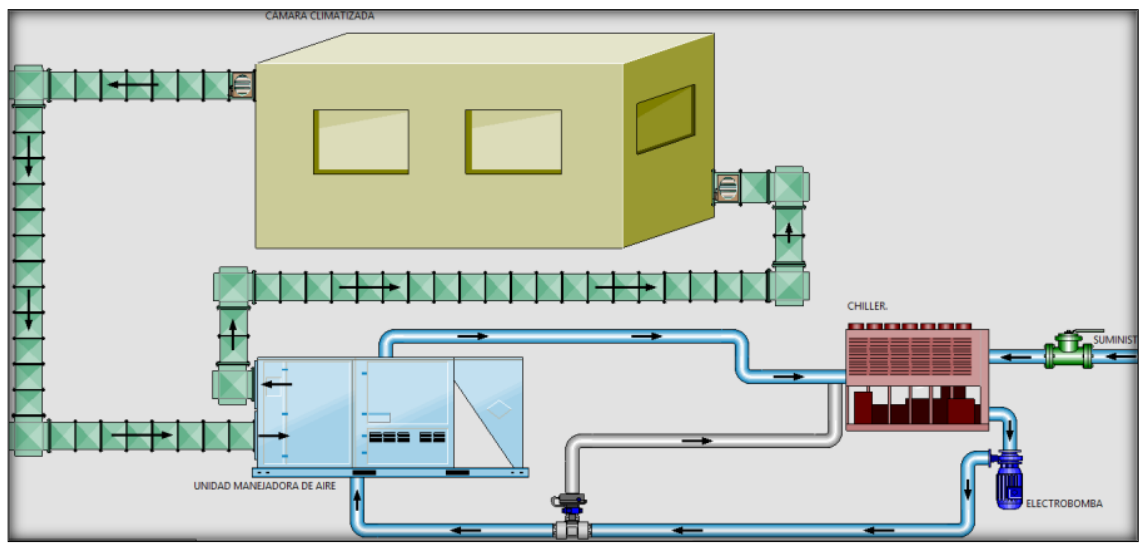

Nota. Imagen tomada de Silva y Soto (2019).

La finalidad principal de la cámara climatizada era generar condiciones de atmósfera controlada para pruebas en equipos de refrigeración domésticos y comerciales. En la automatización, también se ejecutó un componente para que el mismo sistema de supervisión pudiera analizar si de acuerdo con las pruebas realizadas a los equipos, estos aprueban o no la comercialización nacional e internacional. En la Figura 10, se ilustra este componente adicional a la programación para las pruebas de consumo energético.

Figura 10

Componente adicional de aprobación de pruebas de consumo energético
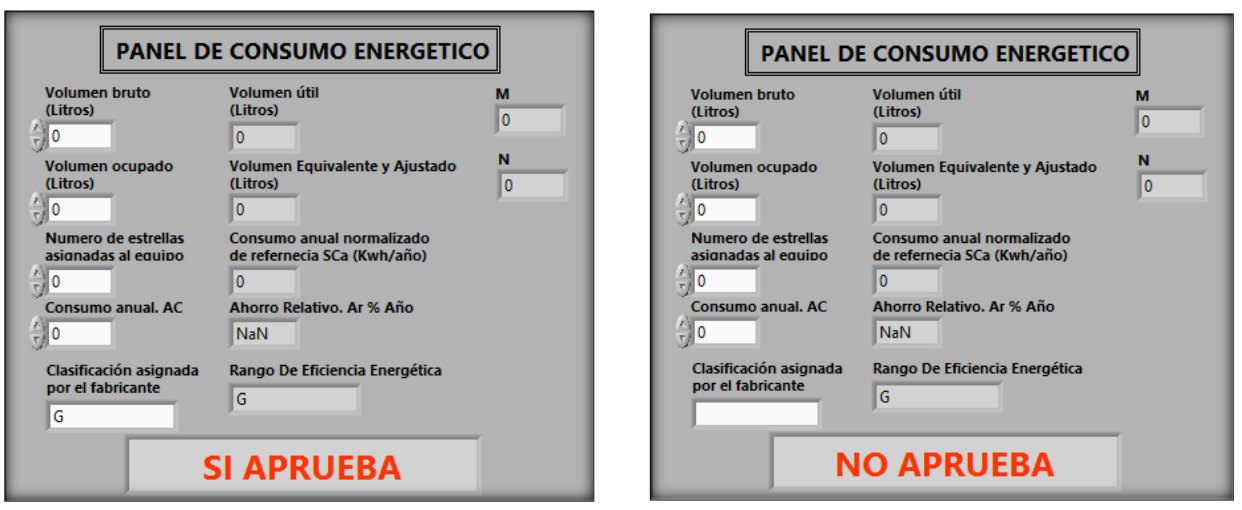

Nota. Imagen tomada de Silva y Soto (2019). 


\section{CONCLUSIONES}

La evaluación de los requerimientos de instrumentación y control permitió hallar la posición, cantidad y características de los instrumentos necesarios para llevar a cabo el objetivo general de la automatización de la cámara climatizada.

Por medio de la ejecución de un modelo matemático, se logró la simulación de los procesos generados por los equipos climatizadores con el fin de adquirir datos importantes para la selección de instrumentos y estrategias de control. El parámetro de mayor relevancia hallado con el modelo matemático fue el tiempo de variación de temperatura de $1^{\circ} \mathrm{C}$ en los laboratorios, que fue de $16,89 \mathrm{~min}$, parámetro que condiciona el tiempo de reacción que deben tener los instrumentos de medición, control y el software de supervisión.

El diseño del sistema de supervisión y control en el software seleccionado permitió simular el control de condiciones atmosféricas generadas en los laboratorios por los equipos de climatización, a través de la configuración, la estrategia de control y la verificación de los distintos comportamientos termodinámicos presentes en las pruebas de consumo energético y de materiales.

\section{Referencias}

Babu, A., Kumaresan, G., Raj, V., y Velraj, R. (2018). Review of leaf drying mechanism and inflencing parameters, drying method, nutrient preservation, and mathematical models. Renewable and Sustainable Energy Reviews, 90, 536556.

Barbosa, S., y Gamboa, J. (2019). Desarrollo de la ingeniería de detalle de la cámara climatizada para pruebas de equipos y materiales. (Trabajo de grado). Universidad de América.

https://repository.uamerica.edu.co/bitstream/20.500.11839/7318/1/413 1688-2019-1-IM.pdf

Cengel, Y., y Boles, M. (2017). Termodinámica. Mc Graw Hill.

Creus S., A. (2010). Instrumentación industrial. AlfaOmega Grupo Editor.

Derazo López, A. (2014). Diseño de la automatización para el control de un chiller de 60 toneladas marca York en la empresa Manufacturas Técnicas Climatizadas. (Trabajo de grado). Universidad Autónoma de Occidente.http://red.uao.edu.co:8080/bitstream/10614/7023/1/T05106. pdf

Enríquez Harper, G. (2000). El abc de la instrumentación en el control de procesos industriales. Limusa.

Instituto Colombiano de Normas Técnicas (ICONTEC), (2004). Norma Técnica Colombiana 5310. ICONTEC. https://docplayer.es/53290635-Normatecnica-colombiana-5310.html 
Instituto Colombiano de Normas Técnicas (ICONTEC), (2011). Norma Técnica Colombiana 5891. ICONTEC. https://docplayer.es/50373568-Normatecnica-colombiana-5891.html

Ministerio de Minas y Energía (MINMINAS). (2014). Anteproyecto Anexo General Reglamento Técnico de Etiquetado. RETIC. MINMINAS. https://www.minenergia.gov.co/documents/10180/794708/Anteproyec toAnexoGeneralRETIQVDefinitiva13Nov2014.pdf/b5658820-60434758-a07f-fba8e34f969e

Muñoz, J. (2018). Diseño de una cámara climatizada, automatizada, con manejo de información por telemetría y condiciones operativas controladas para pruebas de equipos y materiales. (Trabajo de grado). Universidad de América.

https://repository.uamerica.edu.co/bitstream/20.500.11839/6651/1/411 1349-2018-1-IM.pdf

Pérez G., M. A.; Álvarez A., J. C., y Campo R., J. C. (s. f.). Instrumentación electrónica.

Silva, J., y Soto, M. (2019). Diseño de la automatización de la cámara climatizada para pruebas de equipos y materiales. (Trabajo de grado). Universidad de América.

https://repository.uamerica.edu.co/bitstream/20.500.11839/7330/1/413 2728-2019-1-IM.pdf 